\title{
Generation of kitten states using post selection techniques and weak measurements
}

\author{
Sarvraj Singh ${ }^{1}$, P. K. Panigrahi ${ }^{2}$ \\ ${ }^{1}$ Jaypee University Of Engineering And Technology, M.P, India \\ ${ }^{2}$ Indian Institute Of Science Education And Research,Kolkata, India
}

Email address:

sarvraj.5@hotmail.com (S. Singh)

To cite this article:

Sarvraj Singh, P. K. Panigrahi. Generation of Kitten States Using Post Selection Techniques and Weak Measurements, Science Research. Vol. 1, No. 1, 2013, pp. 9-11. doi: 10.11648/j.sr.20130101.12

\begin{abstract}
Using a Cat state as the pointer state of the macro system or the measuring device and a pre and a post selected state of the micro system with common eigenstates. We show that traces of four fold kitten state can be found by spatial distribution of the final device states. Towards the end we elucidate techniques in order to generate a cat state both physically and algebraically with an unknown pointer state.
\end{abstract}

Keywords: Cat State, Weak Measurements,Pointerstates,Kitten States

\section{Introduction}

The idea of Weak Measurements was initially given by aharanov,Albert and Vaidman (AAV) in 1988[1].For a weak interaction between the microsystem and the measuring device, the macrosystem,they show that the measurement results can be much larger than the eigen values of respective observables by correctly selecting the initial and final state of the system .By these measurements results one is able to infer the behaviour of microsystems. This theoretical observation has been shown in various experiments as rotation of photon polarization[2],spin hall effect of light[3]; Weak measurements implemented for high fidelity measurements used by Dixon etal[4-5]to detect very small transverse beam reflection.

The interaction between the measuring apparatus and system under consideration is quite weak, such that linear approximation up to few orders of coupling parameter suffices to depict the interaction, such that coherence in the microsystem remains intact.

The system and measuring apparatus is $H_{\mathrm{int}}=-g(t) A q$, where coupling strength $\int^{t} g(t) d t=g_{o}$ the Unitary evolution

$$
U\left(t_{i}, t_{f}\right)
$$

definedas $\exp -i\left(\int_{t i}^{t} \mathrm{H}(t) d t\right) \Leftrightarrow \exp \left(-\mathrm{g}_{o} A \frac{\partial}{\partial p}\right)$.

Thus if initial state of the system is eigenstate of A ob- servable,such that $A \varphi_{i}(y)=a_{i} \varphi_{i}(y)$. Then

$\hat{U}\left(t_{i}, t_{f}\right) \varphi_{i}(\mathrm{y}) \psi(\mathrm{p})=\varphi_{i}(\mathrm{y}) \exp \left(-g_{o} a_{i} \frac{\partial}{\partial p}\right) \psi(\mathrm{p})=$ $\varphi_{i}(\mathrm{y}) \psi\left(\mathrm{p}-\mathrm{g}_{\circ} \mathrm{a}_{\mathrm{i}}\right) \rightarrow \varphi_{i}(\mathrm{y}) \delta\left(p-\left(p_{o}-g_{\circ} a_{i}\right)\right)$, we will be able to read off value $a_{i}$ unambiguously provided

$\left(\psi\left(\mathrm{p}-\mathrm{g}_{\mathrm{o}} \mathrm{a}_{\mathrm{i}}\right), \psi\left(\mathrm{p}-\mathrm{g}_{\mathrm{o}} \mathrm{a}_{\mathrm{i}}\right)\right) \approx 0$, that is initial spread in momentum is very less than change in momentum . To study intrinsically the disturbance in device pointer's states, it is necessary to select a particular ensemble aptly called post selection,An observable's weak value can be witnessed by a successful post selection.

A quantum weak value could be put as a conditional average of ideally weak measurements ,catering to which a weak value can be strange and lie outside the eigen values of observable,strange weak can be used to amplify a signal,amplification of which lowers technical noise[6].

The pointer of a measuring apparatus is fundamental to quantum measure theory because the values of measures observables are determined from it's properties, use of post and pres selection techniquesintroduces by Schrodinger more than 75 years ago[7][8],PPs techniques have found utility in wide areas of studyas quantum systemenvironment interactions,[9] quantum eraser[10].Studies have been carried onpointer shifts irrespective of the coupling strength between the system and the measuring apparatus.[11].There have been intense works on generation of entangled compass states with sub-planck structures[12].

In this paper we make use of a modified pointer state and analyze the generation of kitten states.

This paper is organised as follows, In section 1 we will give the general shifts of pointer using Gaussian pointer 
state. Section II deals with initialising cat state as initial pointer state, Section III deals with generationof the cat state for Section II implementation

\section{Section I}

In this section we derive expression for device state .Interaction Hamiltonian between the system and measuring apparatus is $H_{\text {int }}=-g(t) A q$, where Ais a dimensionless operator with unit norm,completeeigen states of observable A are $\left|a_{m}\right\rangle$, corresponding eigen values $\left\{a_{m}\right\}$, preselected state $|\psi\rangle=\sum_{m} \alpha_{n}\left|a_{n}\right\rangle$, overall state of system and measuring device is $|\psi\rangle=\sum_{m} \phi_{n n}\left|a_{n}\right\rangle|\phi\rangle$, after evolution $\hat{U}\left(t_{i}, t_{f}\right)\left|\psi_{\mathrm{i}}\right\rangle|\phi\rangle$ where $\hat{U}\left(t_{i}, t_{f}\right)=\exp \left(-\mathrm{i} \int_{t i}^{t /} \hat{H}(t) d t\right) \approx \exp \left(-g_{\circ} \hat{A} \partial / \partial p\right)$,overall state evolves into,

$$
\left|\psi^{\prime}\right\rangle=e^{-i \int H d t}|\psi\rangle=\sum_{m} \alpha_{m} e^{i g a_{m} \Delta}|\mathrm{a} \mathrm{m}\rangle|\phi\rangle
$$

post selected states can be written as

$$
\left|\psi_{f}\right\rangle=\sum_{m} \beta_{m}|\mathrm{am}\rangle
$$

After post selection final device state is given as

$$
\left|\phi^{\prime}\right\rangle=\left\langle\psi_{f} \mid \psi^{\prime}\right\rangle \sum_{m} \alpha_{m} \beta_{m} * \mathrm{e}^{i g a_{m} \Delta}|\phi\rangle
$$

Where $\Delta$ is variable of measuring device.Here initial state of pointer device is assumed to be a Gaussian wave function centered on $\mathrm{q}=0$ and $\mathrm{p}=0$,

Where ' $q$ ' and ' $p$ ' signify the position and momentum representation respectively.

In position representation the Gaussian pointer representation is:

$$
\phi(q)=\frac{1}{\left(2 \pi \Delta^{2}\right)^{1 / 4}} \exp \left(\frac{-q^{2}}{4 \Delta^{2}}\right)
$$

In momentum representation the Gaussian pointer representation is:

$$
\phi(\mathrm{p})=\frac{\left(2 \Delta^{2}\right)^{1 / 4}}{\pi^{1 / 4}} \exp \left(-\Delta^{2} \mathrm{p}^{2}\right)
$$

Which can be easily found by taking the fourier transform of the position space representation.

For our convenience initialize:

$$
\Delta \mathrm{p}=\frac{1}{2 \Delta}, \Delta q=\Delta
$$

Here we take the initialize state as a Gaussian as the things are symmetrical , an enthusiastic reader can reformulate using log normal distribution, which is not symmetrical along it's centre but rather shows biased distribution.

\section{Section II}

In this section, we improvise upon the initial ter ,state which was earlier a Gaussian peaked at $\mathrm{p}=0, \mathrm{q}=0$, to a cat state ,well known by two Gaussians one centered at $-\mathrm{q}$ and other at $+\mathrm{q}$.

The spatial distribution of cat state, coordinate basis

$$
\begin{gathered}
\left\langle x \mid \pm \alpha^{ \pm}\right\rangle=\psi^{ \pm}(x) \\
\left\langle x \mid \pm i \alpha^{ \pm}\right\rangle=\varphi^{ \pm}(x) \\
\text { Where }\left| \pm \alpha^{ \pm}\right\rangle=\frac{1}{\sqrt{2}}(|\alpha\rangle \pm|-\alpha\rangle)
\end{gathered}
$$

These states do not generically satisfy

Cat state in spatial position representation is given by :

$$
|\phi\rangle=\frac{e^{-\left(x+x_{0}\right)^{2} / 2 \delta^{2}} \pm e^{-\left(x-x_{0}\right)^{2} / 2 \delta^{2}}}{\sqrt{2} \pi^{1 / 4} \delta^{1 / 2}\left[1 \pm e^{-x_{0}{ }^{2} / \delta^{2}}\right]^{1 / 2}}
$$

For a particular set of pre and post selection as :

$$
\begin{aligned}
& \left|\psi_{i}\right\rangle=\frac{1}{\sqrt{2}}\left(e^{-i \frac{\phi}{2}}|\uparrow\rangle+i e^{i \frac{\phi}{2}}|\downarrow\rangle\right) \\
& \left|\psi_{f}\right\rangle=\frac{1}{\sqrt{2}}(i|\uparrow\rangle+|\downarrow\rangle)
\end{aligned}
$$

Where the global phase defined for each state.

The final device state is given by:

$$
\left|\phi^{\prime}\right\rangle=\left\langle\psi_{f} \mid \psi_{i}\right\rangle|\phi\rangle
$$

After weak interaction the device state after post selection as given in previous section:

$$
\frac{1}{\sqrt{2}}\left\langle\psi_{f}\right|\left(e^{i g a_{m} q}\left(e^{-i \phi / 2}|\uparrow\rangle+i e^{i \phi / 2}|\downarrow\rangle\right)|\phi\rangle\right.
$$

Solving further for final device state:

$$
\frac{1}{2} i\left(e^{i g q / 2} e^{i \phi / 2}-e^{i g q / 2} e^{-i \phi / 2}\right)|\phi\rangle
$$

The above equation in position representation

$$
\frac{1}{2} i\left(e^{i g q / 2} e^{i \phi / 2}-e^{i g q / 2} e^{-i \phi / 2}\right)\langle x \mid \phi\rangle
$$

The above equation results in two kicks, a couple in position representation and couple in momentum representation .

This confirms the generation of cat states

$$
\frac{1}{2}(|\alpha\rangle+|-\alpha\rangle+|i \alpha\rangle+|-i \alpha\rangle)
$$




\section{Section III}

In the previous section we used a well defined cat state .In this section we derive a way to generate a cat state by simple manipulation and algebraic properties. For a random initial pointer state that shall end up with the cat state as a final state of device .Here we calculate the random initial pointer state.

For the same pre and post selection as in the previous section,

$$
\left|\phi_{i}\right\rangle=\int d x|x\rangle \phi_{i}(x)
$$

Therefore,

$$
\frac{d\left(\left\langle x \mid \phi_{i}\right\rangle\right)}{d x}=\phi_{i}(x)
$$

The final state now is a cat state and we intend to calculate the unknown initial pointer state as witnessed below

$$
\begin{aligned}
& \frac{e^{-\left(x+x_{0}\right)^{2} / 2 \delta^{2}} \pm e^{-\left(x-x_{0}\right)^{2} / 2 \delta^{2}}}{\sqrt{2} \pi^{1 / 4} \delta^{1 / 2}\left[1 \pm e^{-x_{0}{ }^{2} / \delta^{2}}\right]^{1 / 2}} \\
= & \frac{d\left(\left\{i e^{-i \phi / 2} e^{i g q / 2}+e^{-i \phi / 2} e^{-i g q / 2}\right\}\left|\phi_{i}\right\rangle\right)}{d q}
\end{aligned}
$$

Here $\left|\phi_{i}\right\rangle$ is the initial pointer state of the measuring apparatus. Also the $\mathrm{q}$ and the $\mathrm{x}$ represents the position spatial representation.

$$
\frac{\int d x\left(\frac{e^{-\left(x+x_{0}\right)^{2} / 2 \delta^{2}} \pm e^{-\left(x-x_{0}\right)^{2} / 2 \delta^{2}}}{\sqrt{2} \pi^{1 / 4} \delta^{1 / 2}\left[1 \pm e^{-x_{0}{ }^{2} / \delta^{2}}\right]^{1 / 2}}\right)}{\left\{i e^{-i \phi / 2} e^{i g q / 2}+e^{-i \phi / 2} e^{-i g q / 2}\right\}}=\left|\phi_{i}\right\rangle
$$

If we initialize the initial pointer state of the measuring device as one above, for various parameters ' $\mathrm{g}$ ' coupling strength of the interaction and the global phase angle, we are likely to get a cat state.

\section{Conclusion}

Provided the given post and pre selected states, can be synthesized using optics, the generation of quantum kitten states would be quite lucid,subject to conditions that the interactions considered are weak enough such that it doesn't distort the system.

\section{Acknowledgements}

Sarvraj Singh acknowledges the support from the department of Physical Sciences IISER-Kolkata,during the course of this work.

\section{References}

[1] Y. Aharonov, D.Z. Albert, and L. Vaidman, Phys. Rev. Lett., $60,1351(1988)$

[2] KwiatP,Steinberg A and Chio R 1992 Phs. Rev. A 457729

[3] O.hosten and P Kwiat, Science 319,787(2008)

[4] P.B.Dixon,D.J.Starling,A.N.Jordanand J.C Howell,Phys Rev Lett.102,173601(2009)

[5] D.J Starling,P.B.Dixon,A.N.Jordan and J.C Howell,Phys Rev A 80,041803

[6] ]Atsushi Nishizawa and Masa Katsu Fujinoto, P.R.A $85,062108(2012)$

[7] Schrodinger E[1935 Proc.Camb.PhilSoc 31555

[8] Schrodinger E 1936 Proc.Camb Phil Soc 32446

[9] Reznik B 1995 arxiv:quant-ph/9501023v1

[10] Zhang, Shengshi Pang, ChangQiao, Shengun Wu PRA84, 052111(2011)

[11] A.K.Pan and Prasanta.K.Panigrahi,joint weak value for all order coupling

[12] SayanChoudhary, PrasantaK. Panigrahi arxiv:1004.263v2 [quant-ph] 20Apr 2010 